Nervenarzt 2015 - 86:491-491

DOI 10.1007/s00115-014-4243-z

Online publiziert: 20. Februar 2015

(c) Springer-Verlag Berlin Heidelberg 2015
S.G. Meuth

Klinik für Neurologie, Universitätsklinikum Münster

\section{Zeigen die neuen oralen Therapien Vorteile in der Basistherapie der Multiplen Sklerose? Pro}

jeweils auf einer Vorläufersubstanz, sodass neben den Zulassungsstudien bereits weitere Sicherheitsaspekte abgeschätzt werden und entsprechende Programme zum Monitoring aufgelegt werden konnten. Für Dimethylfumarat bestätigte die Extensionsstudie ENDORSE das im Rahmen der Kernstudie beobachtete Sicherheitsprofil, und der kürzlich bekannt gewordene Fall einer progressiven multifokalen Leukenzephalopathie ist darauf zurückzuführen, dass trotz adäquatem Monitoring das Präparat bei einem Patienten mit langanhaltender, ausgeprägter Lymphopenie nicht abgesetzt wurde. Teriflunomid zeigte in den beiden Phase-III-Studien TEMSO und TOWER ein sehr gutes Sicherheitsprofil, welches auch in der Zeit nach der Zulassung bestätigt werden konnte.

Zahlreiche Studien bzw. Ärzte- und Patientenbefragungen zur Adhärenz bei injizierbaren Basistherapeutika haben in den letzten Jahren zeigen können, dass der Prozentsatz adhärenter Patienten als suboptimal einzustufen ist. Unter den am häufigsten angegebenen Gründen für Nichtadhärenz wurde bei $>30 \%$ der Patienten mindestens ein injektionsbezogener Grund (z. B. lokale Reizung, Abneigung vor Injektionen) angegeben [3]. Hier könnten die neuen oralen Therapieformen zu einer deutlich höheren Adhärenz beitragen, und wie sehr die Patienten auf diese Applikationsformen gewartet haben, hat sich unmittelbar nach der Zulassung und im weiteren Verlauf deutlich gezeigt. Dieser Eindruck bestätigte sich auch in einer Vergleichsbefragung zur Zufriedenheit bei Patienten im direkten Vergleich einer Spritzentherapie (Interferon- $\beta$ ) mit Teriflunomid.

\section{Fazit für die Praxis}

Die Neuzulassung der oralen MS-Therapeutika (Teriflunomid - Aubagio ${ }^{\oplus}$, Dimethylfumarat - Tecfidera ${ }^{\oplus}$ ) hat die Versorgungsqualität in der Behandlung der Multiplen Sklerose deutlich erhöht. Den behandelnden Ärzten stehen nun weitere effektive und sichere Optionen im Sinne einer immer individualisierter durchführbaren Therapie zur Verfügung.

\section{Korrespondenzadresse}

Prof. Dr. Dr. S.G. Meuth

Klinik für Neurologie,

Universitätsklinikum Münster

Albert-Schweitzer-Campus 1, 48149 Münster

sven.meuth@ukmuenster.de

Interessenkonflikt. S.G. Meuth hat Honorar für Vorträge, Reisekostenerstattungen sowie finanzielle Unterstützung bei Forschungsprojekten von folgenden Firmen erhalten: Bayer, Bayer Schering, Biogen Idec, Genzyme, Merck Serono, MSD, Novartis, Novo Nordisk, Sanofi-Aventis und Teva.

\section{Literatur}

1. O'Connor P, Wolinsky JS, Confavreux C et al (2011) Randomized trial of oral teriflunomide for relapsing multiple sclerosis. N Engl J Med 365:12931303

2. Havrdova $E$, Hutchinson M, Kurukulasuriya NC et al (2012) Oral BG-12 (dimethyl fumarate) for relapsing-remitting multiple sclerosis: a review of DEFINE and CONFIRM. Expert Opin Pharmacother 14:2145-2156

3. Devonshire V, Lapierre Y, Macdonell R et al (2011) A multicenter observational study on adherence to disease-modifying therapies in patients with relapsing-remitting multiple sclerosis. Eur J Neurol 18:69-77 werden muss, dass der Referenzarm mit GA nicht für einen direkten statistischen Vergleich gepowert war [2].

Die Entwicklung beider neu zugelassenen oralen Therapiealternativen basiert 